\title{
La constitución de un nuevo sujeto social indígena: pueblo guaraní, educación formal y capital intercultural
}

\author{
Yamila Irupé Nuñez \\ ORCID: 0000-0002-0810-7123
}

\section{Resumen}

El ingreso de estudiantes guaraníes en Instituciones de Educación Superior (IES) es un fenómeno reciente tanto en Brasil como en Argentina. En tanto miembros de un grupo que ocupa una posición dominada del espacio social, los guaraníes tienen escasas posibilidades de acceder y permanecer en el sistema educativo superior. Su presencia física en estos espacios expone numerosos problemas relativos a las desiguales condiciones de acceso, permanencia y egreso que el sistema educativo formal presenta para las poblaciones indígenas, y posiblemente para otros miembros de los sectores más desheredados de cada país. La presente contribución, realizada en base a un intensivo trabajo de campo antropológico, apunta a profundizar el conocimiento de este fenómeno, las principales causas y procesos que contribuyen a darle forma, las diversas instituciones sobre cuyas esferas de influencia se ve atraído y las experiencias de los estudiantes guaraníes que lo protagonizan. Para ello, apelando a un estilo narrativo, introduciremos las trayectorias educativas de dos guaraníes, uno de la Provincia de Misiones (Argentina) y uno del Estado de Paraná (Brasil), a fin de transmitir algunos indicios de cómo experimentan los estudiantes guaraníes el tránsito por la educación formal de la sociedad dominante.

\section{Palabras clave}

Educación - Capital intercultural - Profesional indígena - Instituciones de educación superior.

1- Universidad Nacional de Misiones (UNaM), Posadas, Argentina. Contacto: yamilairupe88@gmail.com

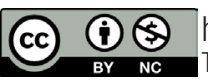




\section{The constitution of a new indigenous social subject: Guarani people, formal education and intercultural capital}

\section{Abstract}

Guarani students' access to Higher Education Institutions (HEI) is a recent phenomenon both in Brazil and Argentina. As members of a group which occupy a dominated position in social space, Guarani people have scarce possibilities of accessing and remaining in the higher education system. Their presence in such places expose numerous issues related to the unequal conditions of access, permanence and completion that the formal education system pose for indigenous populations, and possibly for members of other disinherited social groups in both countries. Based on an intense anthropological fieldwork, this paper aims to further increase our knowledge of this phenomenon, taking into account the experiences of the Guarani students that play a leading role in it, as well as the different institutions engaged throughout the process. By making use of a narrative style, educational trajectories of two Guarani students are presented, one from the province of Misiones (Argentina) and the other from the State of Parana (Brazil), in order to convey some signs of how Guarani students experience the passage through the formal education system of the dominant society.

\section{Keywords}

Education - Intercultural capital - Indigenous professional - Higher education institutions.

\section{Introducción}

A nivel mundial el ingreso y permanencia de estudiantes en instituciones de educación superior (en adelante IES) plantea diversos problemas en lo que se refiere a las desigualdades sociales ante el sistema de educación, y las implicancias de estas desigualdades en los procesos de aprendizaje de los y las estudiantes. En el caso específico de los estudiantes guaraníes de nivel superior (en adelante EGNS) de la Provincia de Misiones (Argentina) y del Estado de Paraná (Brasil), sus posiciones sociales y trayectorias históricas como miembros de un grupo marginado restringen notablemente sus posibilidades de acceso y permanencia en el sistema educativo superior.

El objetivo de esta contribución es mostrar que las políticas recientes -profundamente distintas y temporalmente variables- de incorporación de la población guaraní en el sistema educativo superior de la Provincia de Misiones (Argentina) y del Estado de Paraná (Brasil), conjuntamente con las propiedades sociales de aquellas personas que están mejor dotadas para aprovecharlas, han contribuido a producir la emergencia de un nuevo sujeto social indígena, cuyas características principales serán analizadas a continuación. 
A fin de transmitir algunos indicios de cómo experimentan los estudiantes guaraníes el tránsito por la educación formal, introduciremos las trayectorias educativas de dos guaraníes. Apelando a un estilo narrativo, presentaremos la historia de Aníbal -estudiante avanzado de la Universidad Nacional de Misiones- y de Alex -estudiante guaraní de la carrera de Administración en la Universidade Estadual de Londrina (Brasil)-.

Los resultados aquí presentados fueron producidos a partir de una investigación antropológica, acerca del acceso de la población guaraní al sistema educativo superior. Consideramos que la antropología tiene mucho que aportar a la práctica educativa. A través de la aplicación de una perspectiva comparativa, crítica y desnaturalizadora, la antropología puede asistir al estudio de los procesos educativos en los que intervienen múltiples actores con tradiciones y perspectivas diversas (ROCKWELL, 2009). La potencialidad de nuestra disciplina reside en su capacidad de desnaturalizar categorías de uso cotidiano, a través de un esfuerzo por mostrar las relaciones y procesos históricos que contribuyeron a su producción y aceptación (NEUFELD, 2005). Asimismo, el enfoque antropológico aporta cuestiones que no han terminado de ser tratadas en el campo educativo, "[...] reflexiones que son parte constitutiva de la disciplina en torno a la relación entre educación y cultura, a la noción de producción cultural y a la necesidad de instalar una reflexión sobre los procesos formativos que supere la escolaridad" (NOVARO, 2010, p. 2). En este sentido, este artículo busca generar una reflexión sobre experiencias educativas en contextos interculturales de aprendizaje y enseñanza.

\section{Metodología}

El trabajo de campo a partir del cual se recolectó información se llevó a cabo en comunidades guaraníes de la Provincia de Misiones (Argentina) y en IES donde se registraban estudiantes guaraníes. Para la construcción de la trayectoria educativa de Aníbal, utilizamos entrevistas semiestructuradas y en profundidad, y notas redactadas en conjunto. Es necesario destacar que la reconstrucción de su historia de vida constituye el resultado de varios años de trabajo conjunto. Asimismo, estas entrevistas fueron desarrolladas en contextos más amplios de investigación etnográfica (2007-2012 y 2013-2018).

La información relativa a la situación de la población guaraní y las relaciones con el sistema educativo superior de Estado de Paraná fue producida a partir de una exhaustiva revisión bibliográfica, y a partir de comunicación personal con investigadores y equipos de investigación que estudian la temática en universidades estatales. Roberto Wagner Amaral, profesor investigador de la Universidade Estadual de Londrina, nos brindó datos sobre la situación de los pueblos indígenas en IES de Paraná así como sobre el funcionamiento de los programas destinados a esta población y acceso al registro censal producido por la Comissão Universidade para os Índios (CUIA). Además, mantuvimos comunicación con los integrantes de su equipo de investigación -entre quienes se encuentra Alex-, quienes nos facilitaron información estadística producida sobre la temática allí. Para la reconstrucción de la historia de Alex consultamos el testimonio de su autoría como estudiante indígena en el sistema educativo estatal brasilero (CASSANDRE; AMARAL; SILVA, 2016), de donde tomamos los datos para la interpretación de su trayectoria educativa. 
La elección del caso paranaense responde a varios motivos. En primer lugar, está el hecho evidente de que ambos territorios tienen presencia de población guaraní2 y específicamente mbya-guaraní. Asimismo, en ambas jurisdicciones las universidades públicas constituyen las principales IES. Otro motivo está dado por la cercanía geográfica, dado que Misiones y Paraná comparten frontera. Por último, en ambos territorios se han implementado en años recientes políticas públicas y acciones afirmativas específicamente diseñadas para atender la incorporación de la población indígena en la educación formal (en sus diferentes niveles educativos).

\section{Datos sobre el pueblo guaraní en Misiones y Paraná}

Según el Equipo Mapa Guaraní Continental (EMGC, 2016), los guaraníes constituyen uno de los pueblos indígenas de mayor presencia territorial en el continente americano. El territorio ocupado por este grupo étnico es anterior a la creación y conformación de los actuales Estados-nación y sus fronteras. Con una población mayor a 280.000 personas, el pueblo guaraní tiene presencia demográfica en Argentina, Bolivia, Brasil y Paraguay. En el caso de Argentina, los guaraníes habitan dos regiones bien diferenciadas: la del litoral, especialmente en la provincia de Misiones, y la de noroeste, con mayor presencia en las provincias de Salta y Jujuy.

La provincia de Misiones se sitúa en el noreste argentino (NEA). Se caracteriza por una importante diversidad cultural y lingüística debido a su ubicación en la región de Triple Frontera (Argentina-Brasil-Paraguay), a una política de colonización y de desarrollo agrícola impulsada por el Estado a partir de 1980 -que tenía por objetivo llenar el vacío poblacional en el territorio-, y la presencia de población originaria guaraní, así como de grupos afrodescendientes, previamente radicados allí. Según el criterio de auto-adscripción, los mbya-guaraní constituyen la etnia guaraní representada mayoritariamente ${ }^{3}$; la población guaraní asciende aproximadamente a los 10.000 habitantes distribuidos en 120 tekoa ${ }^{4}$, de los cuales 14 superan los 150 habitantes y sólo uno tiene más de 1.000 habitantes (EMGC, 2016). Mientras que en el Estado de Paraná residen cerca de 25.925 personas que se autoidentifican como indígenas (BRASIL; IBGE, 2010), de las cuales 4.000 se reconocen como perteneciente al pueblo guaraní (FAUSTINO, 2012). Allí, gran parte de la población guaraní es empleada en trabajos rurales en haciendas vecinas en tareas de limpieza o roza, y en las ciudades, en trabajos de baja calificación tales como: albañilería, servicio doméstico, cuidado infantil, etc. Una ocupación importante al interior de las comunidades, aunque de limitadísimo acceso, lo constituyen los trabajos de agente sanitario y profesor bilingüe en las escuelas. A este último, además, solo pueden acceder aquellos/as que están inscritos en

2- Entre los guaraníes la migración es una práctica recurrente que supone un desplazamiento constante por los territorios de Paraguay, Argentina y Brasil.

3 - También residen en sus territorios algunos miembros que se reconocen como chiripá (avá katú eté, también designados como ñandéva) y paí tavyterá (también designados como kayová o pañ) (GOROSITO KARMER, 2006).

4 - También grafiado como tekoha. Es un concepto complejo que constituye la estructura básica de la sociedad guaraní y se conforma por agrupaciones comunitarias organizadas espacialmente por familias extensas. El término deriva de tekó, entendido como "modo de ser/estar, ley, costumbre, hábito" (RUIZ DE MONTOYA, 1876, p. 363), es decir, el tekoa no implica únicamente el espacio físico donde viven los guaraníes, sino que comprende un cierto modo de ser y vivir en dicho lugar. 
el Proceso Selectivo Simplificado ${ }^{5}$ (PSS). Esto ha dado lugar a la reciente configuración de un nuevo "[...] circuito de trabajo indígena intra y/o inter-aldeas" (AMARAL, 2010, p. 437), por el ingreso al mercado de los y las jóvenes indígenas que están insertos en el sistema educativo superior o que han egresado del mismo.

En el caso de la Provincia de Misiones, la principal fuente de ingresos monetarios entre los guaraníes proviene de la venta de artesanías, seguido por el rubro empleado rural y los denominados changarines que realizan empleos temporarios en pequeñas chacras (carpidas, macheteadas, raleo, entre otras) y en plantaciones forestales (desmonte). Dentro de las comunidades guaraníes, aquellos que poseen algún grado de nivel educativo formal, así como las relaciones sociales necesarias, pueden acceder además a los empleos de agente sanitario y/o auxiliar docente indígena (en adelante ADI).

Para esta etnia, el territorio es un elemento fundamental para la conformación del tekoa, su modo de organización sociocultural, y la mantención de su mbya reko (modo de vida). Como mencionamos, la base de este modelo de organización es la familia extensa. Ésta se constituye por grupos macro familiares que ocupan espacios dentro de los territorios guaraní, con base en relaciones de afinidad y consanguinidad. La familia extensa constituye la unidad básica de producción, reciprocidad y consumo. La organización social de los guaraníes se basa en la transmisión de conocimientos -valores religiosos y morales - a través de las relaciones familiares en sus tekoa.

A través de varias generaciones, los guaraníes han sabido transmitir sus prácticas y repertorios culturales con notable eficacia por medio de estrategias propias tales como la oralidad -mediante largas horas de conversación-, la participación activa y temprana de los individuos en las actividades del grupo, rituales, entre otras. La palabra es la equivalencia del alma y ese es el valor otorgado en las relaciones humanas y espirituales una vez distribuidas y reproducidas (ARCE, 2011). De manera que la educación guaraní, basada en la oralidad y en largos rituales, también incorpora la experiencia de todas las etapas de la vida. A través de las palabras que va escuchando y diciendo, y a través de la propia experiencia, la persona guaraní se va consolidando desde su nacimiento, con el acompañamiento de su grupo familiar y su comunidad (LARRICQ, 1993; MELIÀ, 2008). Pero si bien la educación guaraní reproduce lo que los adultos reconocen como su sistema -su propio modo de vida-, esto ocurre sólo en la medida en que el medio y las condiciones de vida permitan la realización del mismo. En este sentido, todas las formas de la educación guaraní están finamente entretejidas con la práctica social circundante (LARRICQ, 1993). Es por ello, que la expropiación de sus territorios supone un ataque directo a su cultura.

Al igual que en el resto de Brasil, la situación demarcatoria de los territorios indígenas en el Estado de Paraná es precaria, pues no se realizan ampliaciones territoriales acordes al aumento poblacional. Como en los demás países de América Latina, las poblaciones indígenas allí constituyen uno de los segmentos más desfavorecidos desde el punto de vista económico, habitacional, educacional y sanitario. De manera similar a lo que ocurre en Argentina, los guaraníes en Brasil sufren un proceso de expropiación territorial que

\footnotetext{
5- Sistema de selección realizado por la Secretaria de Educación del Estado de Paraná (SEED) para conformar el cuadro de profesores. El proceso se realiza sólo a través de documentos comprobatorios de formación, sin la necesidad de una selección a través de aplicación de prueba (AMARAL, 2010). Sin embargo, sólo se otorga el cargo si los documentos están acompañados de una Carta de Anuência del cacique, autorizando a la persona a ocupar la vacante, independientemente del orden de clasificación en que se encuentre.
} 
se intensificó a partir de mediados del siglo XX, fuertemente motivado por la injerencia de las élites agrarias. Más aún, actualmente la realidad paranaense en la demarcación de tierras y reconocimiento de derechos de los pueblos indígenas experimenta un retroceso sobre las conquistas históricamente alcanzadas.

El deterioro en la calidad de vida de los guaraníes se ha venido acrecentando, y se percibe a través de la creciente instalación de pequeños grupos familiares en viviendas precarias al borde de las principales rutas, generalmente construidas con materiales de desecho. También se ha intensificado su instalación en las periferias de ciudades y pueblos sin acceso a servicios públicos, en los que los niños y las mujeres recorren las zonas céntricas practicando la mendicidad. Las familias que aún se mantienen en zonas rurales, rodeadas de propiedades que han eliminado las masas boscosas, carecen de espacio suficiente para realizar cultivos de subsistencia y sufren la disminución alarmante de los recursos de la fauna y la flora con las que décadas atrás resolvían sus necesidades. De esta forma, se ven forzados a compensar estas pérdidas con un consumo creciente de productos provenientes del intercambio mercantil o de la asistencia alimentaria estatal.

El proceso de ocupación y transformación del territorio por sectores dominantes de la sociedad tiene una incidencia directa en las costumbres del pueblo guaraní. En diversos períodos históricos, las poblaciones guaraníes han tenido que cambiar sus estrategias económicas con el objetivo de garantizar ingresos que les permitieran suplir prácticas y recursos cuyo acceso fue creciente y sistemáticamente negado. Sin embargo, su posición desventajada en el espacio social los obliga a incorporarse en los puestos peor remunerados del mercado de trabajo rural, en distintos tipos de actividad. Asimismo, estas transformaciones repercuten en el tipo de estrategias que el Estado diseña para relacionarse con dicha población. Especialmente desde el punto de vista de las políticas públicas que toman a los grupos indígenas como objeto, así como en las estrategias de resistencia étnica que los propios guaranies generan, siendo una de ellas la apropiación de la educación formal de la sociedad dominante.

En los últimos años la centralidad de la escuela se ha hecho extensiva a la educación superior, que es la que asegura el acceso a ocupaciones más o menos bien remuneradas, como antaño lo hacía la educación secundaria. Por otra parte, la opción por la educación superior tiene el potencial de convertirse en una oportunidad no sólo para insertarse en un mundo laboral y profesional más amplio, sino también para disponer de autonomía de pensamiento, y manejar herramientas conceptuales y técnicas para contribuir con el desarrollo y la creación del sistema educativo de calidad que las poblaciones indígenas de Misiones demandan. En efecto, la extensión de la educación entre los varones y mujeres indígenas constituye así la última manifestación del proceso de modernización y expropiación impulsado desde la sociedad dominante, y se presenta como una estrategia clave para resistir, mantener y reivindicar la propia cultura. Desde la perspectiva de una auxiliar docente indígena mbya-guaraní:

Me entusiasma mucho que muchos chicos sigan estudiando. Creo que la globalización está muy avanzada. Y nosotros, a medida que el mundo mismo va cambiando, creo que también necesitamos. Para adaptarnos bien en ese mundo, y en las cosas que van cambiando. Lamentablemente hay que hacer cosas para que no sea tan chocante nuestra cultura. Lo único que queda es hacer eso: estudiar y saber algo más. (Mujer, estudiante y ADI - tekoa Peruti). 
Sin embargo, las estadísticas nacionales indican que los guaraníes de Misiones son uno de los grupos étnicos con mayores índices de analfabetismo del país, con el 29,34\% (ARGENTINA; INDEC, 2004/2005). Hace más de una década, la Encuesta Complementaria de Pueblos Indigenas ${ }^{6}$ (ECPI) informaba que para una población de 2.498 personas de ambos sexos de 10 años y más, había 1.763 personas con conocimientos de lecto-escritura en castellano y 733 analfabetos (el 25\% aproximadamente de la población considerada). En cuanto a las personas de 15 años de edad o más que declaraban esos conocimientos, el máximo nivel alcanzado era secundario incompleto (209 personas, aproximadamente el 10\% del grupo alfabetizado), en proporciones similares para ambos sexos.

Tabla 1- Población mbya-guaraní de 10 años o más por sexo según condición de alfabetismo. Misiones. Años 2004-2005

\begin{tabular}{c|ccccc}
\hline SEXO & ALFABETOS & ANALFABETOS & TOTAL \\
\hline FEMENINO & 776 & 447 & 1224 \\
\hline MASCULINO & 987 & 1763 & $\vdots$ & 733 & 1274 \\
\hline TOTAL & $\vdots$ & 1768 & 2498 \\
\hline
\end{tabular}

Fuente: Elaboración propia con base en datos de (ARGENTINA; INDEC, 2004/2005).

Tabla 2- Población mbya-guaraní de 15 años o más por sexo según máximo nivel de instrucción alcanzado. Misiones. Años 2004-2005

\begin{tabular}{|c|c|c|c|c|c|}
\hline SEXO & SIN INSTRUCCIÓN & PRIMARIA INCOMP. & PRIMARIA COMPL. & SECUNDÁRIA INCOMP. & TOTAL \\
\hline FEMENINO & 402 & 318 & 119 & 195 & 981 \\
\hline MASCULINO & 271 & 384 & 216 & 104 & 1031 \\
\hline TOTAL & 673 & 702 & 335 & 209 & 2012 \\
\hline
\end{tabular}

Fuente: Elaboración propia con base en datos de (ARGENTINA; INDEC 2004/2005).

En el año 2007, un relevamiento realizado por personal del Proyecto Educación Intercultural Bilingüe (PEIB) con sede en el Ministerio de Educación y Cultura de la Provincia de Misiones, había detectado cinco jóvenes guaraníes en establecimientos terciarios y universitarios -Facultad de Humanidades y Ciencias Sociales, Instituto de Formación Docente Escuela Normal Mixta "Estados Unidos del Brasil" y Facultad de Derecho-. En respuesta a esta situación, en el año 2008 se presentó desde la Facultad de Humanidades y Ciencias Sociales de la Universidad Nacional de Misiones (FHyCS-UNaM), el Proyecto Oñopytyvô Va'ekuéry Oñemboeápy (Los que ayudan a estudiar) al Programa Nacional de Voluntariado Universitario. Esta propuesta se constituye así en el primer antecedente de acción afirmativa destinada a la población guaraní de Misiones dentro de la UNaM. La iniciativa contemplaba el dictado de tutorías, talleres de lectura, y visitas a las comunidades guaraníes con el fin de divulgar las posibilidades académicas de la universidad. Aunque la propuesta no contó con

6 - Esta encuesta, preparatoria de la pregunta étnica en el Censo Nacional de Población (2010), fue aplicada por el INDEC (Instituto Nacional de Estadística y Censos de la República Argentina) en el período 2004/2005 y administrada en la provincia por la Dirección de Asuntos Guaraníes y por agentes censales indígenas seleccionados por ésta y adiestrados por técnicos del INDEC. 
financiamiento, logró sumar la adhesión de todos los estudiantes guaraníes y de algunos estudiantes no-indígenas de la facultad que se desempeñaron como tutores, entre los cuales nos incluimos. Como resultado directo de esta acción, en 2009 se registra un aumento en las inscripciones de estudiantes guaraníes en el sistema educativo superior de la provincia. Ese mismo año, el proyecto se amplía a toda la Universidad como Programa de promoción y apoyo a estudiantes guaranies que cursan carreras en la UNaM, a través del cual se realizaron reuniones con docentes con el fin de evaluar los planes de estudio de las carreras ${ }^{7}$, y se aseguró que los estudiantes guaraníes pudieran contar con becas de pasajes, comedor, apuntes y un albergue exclusivo. Sin embargo, no se consiguió garantizar una beca de apoyo monetario tanto para ellos como para los tutores (quienes también eran estudiantes), lo que influyó negativamente en la regularidad del acompañamiento tutorial y, consecuentemente, en el progresivo abandono de los tutores y la deserción de los estudiantes guaraníes. A continuación, se detallan los datos de matriculación registrados durante la aplicación de dicho programa entre 2009 y 2012, indicando la situación actual de cada caso en términos de permanencia o deserción:

Tabla 3- Datos de matriculación registrados durante la aplicación de dicho programa entre 2009 y 2012

\begin{tabular}{|c|c|c|c|c|c|c|}
\hline $\mathrm{N}^{\circ}$ & AÑO & COMUNIDAD & $\begin{array}{l}\text { LOCALIDAD DONDE } \\
\text { VA A ESTUDIAR }\end{array}$ & CARRERA & INSTITUCIÓN & ESTADO \\
\hline 1 & 2009 & Chapaí, San Ignacio, Depto. San Ignacio & Posadas & Lic. en Letras & UNaM-FHyCS* & Deserción \\
\hline 2 & 2009 & $\begin{array}{l}\text { Ka'aguy Poty, A. del Valle, Depto. } \\
\text { Cainguás }\end{array}$ & Posadas & Lic. en Antropología & UNaM-FHyCS & Deserción \\
\hline 3 & 2009 & $\begin{array}{c}\text { Yvy Pitá, Ruiz de Montoya, Depto. Lib. } \\
\text { Gral. San Martín }\end{array}$ & Posadas & $\begin{array}{l}\text { Lic. En Comunicación } \\
\text { Social }\end{array}$ & UNaM-FHyCS & Deserción \\
\hline 4 & 2009 & 25 de Mayo, Depto. 25 de Mayo & San Pedro & Tec. en Guarda-parque & ISSP - UNaM** & Deserción \\
\hline 5 & 2009 & Perutí,El Alcázar, Depto. Montecarlo & Posadas & Lic. Trabajo Social & UNaM-FHyCS & Continúa \\
\hline 6 & 2009 & $\begin{array}{l}\text { Marangatú, Capioví, Depto. Lib. Gral. San } \\
\text { Martín }\end{array}$ & Posadas & Guía de Turismo & UNaM-FHyCS & Deserción \\
\hline 7 & 2009 & Perutí, El Alcázar, Depto. Montecarlo & Posadas & Abogacía & $\begin{array}{l}\text { Universidad Católi- } \\
\text { ca de Santa Fé }\end{array}$ & Deserción \\
\hline 8 & 2009 & Perutí, El Alcázar, Depto. Montecarlo & Posadas & Lic. En Turismo & UNaM-FHyCS & Deserción \\
\hline 9 & 2009 & 25 de Mayo, Depto. 25 de Mayo & San Pedro & Tec. en Guarda-parque & ISSP - UNaM & Deserción \\
\hline 10 & 2009 & $\begin{array}{l}\text { Tacuapí, Ruiz de Montoya, Depto. Lib. } \\
\text { Gral. San Martín }\end{array}$ & Posadas & Prof. y Lic. en Historia & UNaM-FHyCS & $\begin{array}{c}\text { Fallece en } \\
2012\end{array}$ \\
\hline 11 & 2009 & Katupyry, San Ignacio, Depto. San Ignacio & Posadas & Lic. en Antropología & UNaM-FHyCS & Deserción \\
\hline 12 & 2009 & Fortín Mbororé, Iguazú, Depto. Iguazú & San Pedro & Tec. en Guarda-parque & ISSP - UNaM & Deserción \\
\hline 13 & 2009 & Fortín Mbororé, Iguazu, Depto. Iguazú & San Pedro & Tec. en Guarda-parque & ISSP - UNaM & Deserción \\
\hline 14 & 2010 & Perutí, El Alcázar, Depto. Montecarlo & Posadas & Lic. Trabajo Social & UNaM-FHyCS & Continúa \\
\hline 15 & 2011 & Posadas, Capital & Posadas & Lic. Antropología Social & UNaM-FHyCS & Deserción \\
\hline 16 & 2012 & , 25 de Mayo, Depto. 25 de Mayo & Posadas & Lic. Antropología Social & UNaM-FHyCS & Deserción \\
\hline
\end{tabular}

${ }^{*}$ Facultad de Humanidades y Ciencias Sociales / Universidad Nacional de Misiones.

** Instituto Superior San Pedro de La Universidad Nacional de Misiones

Fuente: Elaboración propia.

$\overline{7}$ - En especial el contenido relativo al primer año de cursado, pues si bien se concibe que los estudiantes necesitan tanto apoyo económico como académico, también es necesario realizar reformas a los planes de estudio y a las IES en general. Lamentablemente, el Programa no contó con el respaldo suficiente para realizar dicho objetivo. 
En el período 2013-2014 no se registraron en la UNaM inscripciones de estudiantes guaraníes. Este hecho, así como la evidencia de la elevada tasa de deserción, motivó a dos estudiantes guaraníes de Trabajo Social de la FHyCS, a solicitar nuestra colaboración para elaborar un programa de becas y acompañamiento tutorial, que fue presentado en 2014 con el nombre de Jaguata pav Ñembo'eapy (Caminemos todos por la Educación) a la Secretaría de Políticas Universitarias (SPU), y aprobado a fines de ese mismo año. El principal objetivo de este programa era llevar la educación intercultural más allá del nivel secundario y propiciar la inserción de estudiantes guaraníes en el nivel educativo superior de Misiones, tanto universitario como terciario, en IES públicas y privadas.

La aprobación del Programa, con carácter experimental por tres años, tenía el objetivo de apostar por un programa de continuidad, habida cuenta de las profundas asimetrías que existen entre los estudiantes guaraníes en sus trayectorias de escolaridad primaria y secundaria. El programa apuntaba a responder la demanda de los guaraníes de acceso a la educación de nivel superior (universitaria, terciaria e institutos superiores de formación docente) y tenía como objetivo contribuir al aumento de la matrícula de estudiantes guaraníes, su índice de permanencia y egreso, para finalmente poder avanzar sobre el propósito de fondo: revalorizar la cultura guaraní a través de su propia población. A pesar de que el Programa recibió una valoración altamente positiva por parte de todos los grupos involucrados, fue terminado abruptamente a finales de 2015 debido a que dicho enfoque no se consideraba prioritario para la nueva gestión de gobierno nacional. Ante la falta de apoyo económico y tutorial, muchos guaraníes abandonaron sus estudios y regresaron a sus comunidades. El retroceso que supone esta falta de apoyo es inconmensurable. Como impacto directo, muchos de estos jóvenes que formaron parte del programa se verán forzados a dedicarse a tareas de baja cualificación y remuneración (la mayoría de las veces en el sector rural) y probablemente jamás tengan la posibilidad de retomar sus estudios.

En el contexto brasilero, el Estado de Paraná es pionero en lo que respecta al ingreso de indígenas en universidades públicas (BERGAMASCHI; BARCELOS DOEBBER; OLIVEIRA BRITO, 2018). En 2002 se implementó allí una iniciativa inédita ${ }^{8}$ en aras de ampliar los derechos de poblaciones indígenas para acceder a la educación superior, cuyos principales puntos consistieron en: a) la garantía legal de vacantes suplementarias para indígenas en las universidades estatales; b) la garantía de procesos selectivos específicos e interinstitucionales (un vestibular ${ }^{9}$ específico para personas pertenecientes a pueblos indigenas ${ }^{10}$ ); c) la garantía de becas de ayuda para la permanencia de estos/ as estudiantes indigenas; y d) el diseño institucional de acompañamiento a estos/as estudiantes y académicos. A diferencia del caso misionero, esta acción afirmativa ha logrado institucionalizarse, tornándose una política pública de carácter permanente y sistemática. Al tener mayor profundidad temporal y mayor cantidad de egresados, el

\footnotetext{
8 - En el 2001 se implementó el primer programa de reserva de vacantes para estudiantes indígenas en cursos regulares (AMARAL, 2010).

9- El vestibular es un medio de ingreso a las universidades en Brasil. Se trata de una evaluación de los contenidos adquiridos en los niveles fundamental y medio (equivalentes al primario y secundario en Argentina).

10- En Paraná, a través de la Ley $n^{0} 13.134$ se estableció en el año 2001 un vestibular específico para el ingreso de indígenas en las Universidades Estatales de Paraná. Las seis universidades estatales públicas del Estado de Paraná realizan todos los años este vestibular especialmente para las comunidades indígenas paranaenses. Es decir, las vacantes ofertadas son suplementarias a las vacantes regulares.
} 
caso de Paraná constituye una muestra que amplía la que realizamos hasta ahora en el contexto misionero. De allí podemos extraer una serie de aprendizajes para pensar nuestra realidad. Por ejemplo, que un programa de acción afirmativa que produce las condiciones para aumentar el ingreso de las poblaciones indígenas en IES, cuando no es acompañado de transformaciones institucionales suficientes para asegurar la permanencia y especialmente el egreso, resulta insuficiente para garantizar el éxito de esta política en términos de inclusión educativa.

\section{Trayectorias de dos estudiantes guaraníes}

En las narrativas que presentaremos, las familias de los estudiantes y las comunidades en las que forjan sus primeras herramientas educativas se caracterizan por poseer un bajo volumen de capital económico y cultural (en comparación con las de sus compañeros/as de la universidad) en un medio donde las condiciones materiales son sumamente limitadas, lo que incrementa la dificultad para estudiar en el sistema formal, no dejando muchas veces más alternativa que la de abandonar los estudios para obtener un trabajo, usualmente informal y mal remunerado. De esta manera, la opción por la educación formal, en especial de nivel superior, no constituye una estrategia regular y, cuando sí ocurre, es escasamente viable. Aquellos/ as estudiantes guaraníes que sí llegan a IES provienen de familias con posiciones dominantes en el ámbito comunitario y están, por lo tanto, mejor dotados para acceder y capitalizar esta experiencia. Y, una vez insertos, pasan a conformar un grupo aún más selecto a partir de contar con recursos económicos (becas, acceso a trabajos diferenciados), culturales (conocimiento interno de algunas de las principales instituciones de la sociedad dominante, como la universidad) y sociales (una red de contactos personales e institucionales más amplia) superiores e inaccesibles a otros miembros de sus comunidades. Esta batería de recursos que Pöllmann (2016a; 2016b) denomina capital intercultural ${ }^{11}$, otorga a los estudiantes guaraníes de nivel superior mayor reconocimiento que la enorme mayoría de sus pares indígenas.

\section{Aníbal, estudiante guaraní de Misiones}

Aníbal tiene 32 años, y junto a su hermano Martín, son actualmente los únicos estudiantes guaraníes de la Licenciatura en Trabajo Social de la UNaM. Nació en 1986 en una comunidad indígena del municipio de Montecarlo (Provincia de Misiones, Argentina), en el seno de una familia de origen étnico mixto. Su padre, Julio, era un peón rural criollo que se había enamorado de Rosa Benítez, quien provenía de una familia guaraní. La nueva pareja se asienta en la residencia materna en Perutí, comunidad guaraní de 588 hectáreas con título de propiedad comunitario, en la que actualmente residen 160 familias. Allí crían seis hijos, cuatro varones y dos mujeres.

11 - Entendido como "[...] una forma de capital sociocultural que incluye experiencias, habilidades y competencias interculturales [y] como todo tipo de capital cultural, es de carácter simbólico -incluyendo maneras de ser, ver, actuar y pensar-y provee recursos para lograr el éxito más allá de las formas tradicionales de riqueza económica" (PÖLLMANN, 2016b, p. 1). 
Mis padres no fueron al colegio. Mi papá plantaba algunas hectáreas de yerba. Plantaba para consumir y para vender también... en las chacritas ${ }^{12}$ que hacen para la comunidad. Yo me acuerdo que siempre plantaba maíz y había gente de afuera que venía a comprar por bolsa. Cuando yo era un poquito más grande trabajé ahí juntando maíz y plantando mandioca. Y así mi papá tenía pensamiento de 'gente de afuera', porque en la comunidad casi no hay eso de chacra grande así para plantar. Pero él trataba por lo menos de tener dos hectáreas de yerba como para vender.

Durante su infancia, Aníbal asiste junto a sus hermanos y hermanas a la escuela ubicada en dicha comunidad, aunque las diferencias de edad eran importantes, por lo que pronto los mayores fueron formando sus propias familias y abandonando la residencia materna. A los 12 años Aníbal pierde a su padre, a quien lo acompaña tres años después su madre. De esta manera el hogar queda a cargo de sus hermanos mayores, José, de 20 años y Martín, de 17, que todavía no habían conformado familias aparte. Debido a que sus otros hermanos tenían familias propias que mantener, no podían ayudar a sus hermanos menores, por lo que José pasó a ser el sostén económico de la reorganizada unidad doméstica, trabajando como tarefero ${ }^{13}$. Por este motivo tuvo que suspender momentáneamente sus estudios hasta que, gracias a una monja que vivía y enseñaba dentro de la comunidad, pudo obtener una ayuda económica para finalizar su educación formal secundaria en el Sistema Provincial de Teleducación y Desarrollo (SIPTeD), mediante un régimen de enseñanza acelerada que combina modalidades semipresencial y a distancia. Sin embargo, José no sólo utilizaba el recientemente generado ingreso económico para alimentar a Aníbal y a Martín, sino que también lo distribuía entre sus otros hermanos, cuando éstos lo precisaban. De esta manera, Aníbal afirma que "La ayuda económica que gestionaba la Hermana no era suficiente porque dividiamos entre los hermanos que necesitaban". Mientras completaba sus estudios, José continuaba trabajando temporalmente en la tarefa, hasta que consigue incorporarse en la cooperativa de trabajo de Peruti y, a partir de allí, desempeñarse como albañil en diversas construcciones en la cercana ciudad de El Alcázar, lo que significó una mejora económica sustancial para el grupo doméstico.

La trayectoria educativa de Aníbal sintetiza las dificultades que para el pueblo guaraní supone lo que gran parte de la población nacional experimenta como un destino inevitable, ininterrumpido y cuasi natural, a saber, la finalización de los estudios secundarios. En la escuela Instituto Hogar Perutí, Aníbal completó la educación primaria $\mathrm{y}$, mediante la modalidad EGB3 ${ }^{14}$, consiguió terminar el octavo grado (correspondiente al primer año del secundario convencional). Corría el año 2005, y por aquél entonces ese era el último grado educativo que se podía cursar dentro de la comunidad, por lo que para continuar sus estudios secundarios, Aníbal y Martín tenían que caminar diariamente 15 kilómetros ida y vuelta hasta el Bachillerato con Orientación Laboral Polivalente (BOLP)

\footnotetext{
12- Pequeñas chacras.

13- Trabajador informal que se encarga del corte de la hoja de yerba mate y de su recolección. Esta actividad, la tarefa, es realizada por cuadrillas en los campos y se realiza anualmente entre los meses de agosto - septiembre. Constituye uno de los empleos peor remunerados de la provincia, y sus trabajadores los miembros más desprotegidos del mercado laboral.

14- La Educación General Básica (EGB) es el ciclo de estudios obligatorio de 9 años dividido en 3 ciclos: $E G B 1: 1^{\circ}, 2^{\circ}$ y $3^{\circ}$ Año de escolarización. EGB2: $4^{\circ}, 5^{\circ}$ y $6^{\circ}$ Año de escolarización. EGB3: $7^{\circ}, 8^{\circ}$ y $9^{\circ}$ Año de escolarización.
} 
n 19 Gral. Manuel de Rosas, ubicado en El Alcázar. Sin embargo, a mediados de ese año tuvo que abandonar sus estudios debido a la necesidad de trabajar en la tarefa ${ }^{15}$ para complementar el ingreso familiar.

Mi hermano mayor [José] decía que yo tenía que dejar el estudio para colaborar en el trabajo con él, y así también en la compra de mercadería para la casa. En ese momento había mucha demanda de tarefa y casi todos los jóvenes de la comunidad se iban a tarefear. Por eso, mi hermano mayor decía que rendía muy poco y necesitaba de otro que colabore con él. Mi hermano mayor era el responsable de nosotros.

En 2006 intentó nuevamente retomar el noveno grado de EGB3, pero de nuevo tuvo que abandonar el cursado para trabajar. La tarefa constituye una de las peor remuneradas actividades rurales del país, y hasta el día de hoy se paga por tonelada de yerba mate cosechada. En este sentido, la estrategia de su hermano es idéntica a la que realizan cientos de familias rurales que se dedican a esta actividad, que de lo contrario resulta insuficiente para mantener una familia nuclear, mucho menos una extensa como la de Aníbal.

Finalmente, en 2007 Aníbal consigue trasladarse junto a Martín, su primo Fernando y otros nueve jóvenes guaraníes a un albergue ubicado en Dos de Mayo, que había sido gestionado en la Dirección de Asuntos Guaranies a pedido de su tío, que en ese momento era cacique de Perutí. La misma institución gestionaba para ellos pasajes de colectivo para que visitaran semanalmente sus comunidades, aunque la irregularidad de este beneficio hacía que tuvieran que dilatar su retorno a veces por lapsos de hasta un mes. Allí cursó hasta el tercer año del Polimodal ${ }^{16}$ bajo una modalidad acelerada en el Bachillerato $\mathrm{n}^{\circ}$ $42^{17}$, pero faltando apenas cuatros meses para la finalización del año lectivo tuvieron que abandonar sus estudios nuevamente, perdiendo el año completo. En esta ocasión el motivo no fue la necesidad de trabajar, sino el desalojo de su residencia debido a que

La Dirección de Asuntos Guaraníes no pagaba la cuota mensual de alquiler del albergue para nosotros. Éramos aproximadamente 12 estudiantes de tres comunidades: Perutí (El Alcázar), Marangatú (San Ignacio), Ojo de agua (Concepción de la Sierra), El Pocito (Capioví) y algunos de Wanda.

De la docena de estudiantes guaraníes sólo uno pudo terminar el secundario allí. Como alternativa, la institución gubernamental les ofrece un albergue exclusivo para guaraníes ubicado en Posadas, donde podían finalizar sus estudios. "Ese mismo año algunos nos vinimos a Posadas para continuar y terminar el secundario". La convivencia conjunta con otros guaraníes resulta clave en esta etapa, para que el desplazamiento hacia un centro urbano de mayor envergadura y a mayor distancia de sus comunidades no

15- Proceso de corte de la hoja de yerba mate directamente desde la planta, realizado por cuadrillas en los campos donde se cultiva. Esta tarea se realiza anualmente entre los meses de agosto y septiembre.

16- Era el ciclo profesional de la enseñanza de nivel medio en la Argentina. Fue un tipo de educación que se utilizó en el país, actualmente no se encuentra vigente.

17- Es el nombre de la institución donde realizó sus estudios. 
resulte tan dramático. Finalmente, en el año 2008, Aníbal, su hermano y su primo pudieron completar la educación secundaria en el colegio BOLP $n^{0} 1^{18}$. Al igual que otros miembros de la etnia guaraní, la consecución de un título secundario resulta dilatada, cuando no imposibilitada, debido a múltiples factores. La necesidad de incorporarse tempranamente al mercado laboral tiene sin duda un peso determinante, y es el principal motivo por el que la mayor parte de los guaraníes en Misiones no terminan la educación formal. Sin embargo, aun cuando este impedimento pueda ser momentáneamente postergado, también aparece como recurrente la necesidad de desplazamiento lejos de la familia y el grupo de pertenencia, así como la dependencia de algún tipo de financiamiento externo, sea en la forma de ayudas económicas o pagos de albergue, que, en última instancia, dependen intimamente de voluntades político-institucionales o bondades de sujetos individuales.

Con el título secundario en mano, Aníbal regresa a Peruti tras cuatro años de alejamiento. Sin embargo, una vez allí es motivado por sus hermanos y por la misma monja que los había ayudado varios años antes, para comenzar en 2009 una Tecnicatura en Construcción en Madera en el ITEC - Instituto Tecnológico ubicado en la cercana ciudad de Montecarlo.

Había una Hermana que vivía en mi comunidad...ella fue quien me ayudó para seguir estudiando. Gestionó una media beca para pagar la matrícula de inscripción y la otra mitad aportaba la Municipalidad de Montecarlo.

La monja además consiguió gestionar a través de la iglesia pasajes semanales de ida y vuelta para Aníbal, así como mercadería (o bien un monto equivalente a una compra mensual de alimentos) y dinero para alquilar una habitación en la ciudad. No obstante, las distintas ayudas económicas no fueron suficientes para cubrir los gastos de su carrera, pues precisaba de materiales y herramientas (como una computadora) específicas para realizar los planos y trabajos prácticos, no quedándole más opción que interrumpir su formación. Otro de los inconvenientes que influyó en el abandono de la carrera fue la falta de acompañamiento o ayuda de tipo tutorial para la comprensión y guía en sus estudios. Asimismo, la vida en la ciudad era para Aníbal excesivamente distante y distinta ${ }^{19}$ de la vida comunitaria dentro del tekoa.

En el 2009, su hermano Martín ingresó en la carrera de Licenciatura en Trabajo Social de la UNaM. Contagiado por sus comentarios, Aníbal decide en 2010 inscribirse en la misma carrera.

Martín vino acá con mi primo y estaba haciendo la carrera... me comentó qué era Trabajo Social, para qué era y con ese título, en dónde podía trabajar. Martín ingresó antes y todas las

18- Las escuelas y bachilleratos Ilevan números. BOLP significa Bachillerato con Orientación Laboral Polivalente Número 1. También es conocido por su nombre BOLP N1 Combate Vuelta de Obligado (Ciudad de Posadas, Provincia de Misiones, Argentina).

19- Las diferencias no remiten únicamente a lo visual y espacial (las comunidades residen en zonas de selva o rurales) sino también a lo simbólico y cultural. El alejamiento físico y espiritual que implica el abandono momentáneo del mbya reko por períodos más o menos largos y sucesivos, debe ser contrarrestado con procesos y rituales que podríamos denominar de purificación, con el objeto de limpiar el ser del contacto con los Juruá. Es el principal motivo por el que todos los viernes (en algunos casos antes) los y las estudiantes regresan a sus tekoa para mantener las dinámicas familiares y comunitarias, que permiten la reproducción de la lengua materna y pautas culturales propias del pueblo mbya-guaraní. 
dificultades y problemas que tenía me contaba, así yo sabía qué tenía que hacer para no tener esas dificultades... eso me incentivó más para venir y continuar, y a mí me fue un poco mejor.

A poco de iniciar su carrera universitaria, Aníbal tomó contacto con estudiantes de distintas carreras que estaban desarrollando un Proyecto de Voluntariado Universitario con el objeto de dar acompañamiento tutorial a estudiantes guaraníes de la facultad. Junto con la experiencia previa de su hermano, estas tutorías fueron de gran ayuda para su rendimiento académico. El contacto con otros estudiantes avanzados le permitió conocer las becas disponibles para personas de bajos recursos, y una vez que obtuvo la beca del Programa Nacional de Becas Universitarias (PNBU), fue conociendo, concursando y obteniendo otras, como la que otorga $\operatorname{Progresar}^{20}$ o la beca provincial para estudiantes terciarios y/o universitarios pertenecientes a pueblos originarios del Comité Ejecutivo de Desarrollo e Innovación Tecnológica (CEDIT). Durante todo este tiempo su esposa cobraba además la Asignación Universal por sus seis hijos, quienes vivian en Peruti junto con ella. Su mejor momento financiero y académico, sin embargo, fue durante el lapso que funcionó el Programa Caminemos todos por la educación, financiado por la Secretaría de Políticas Universitarias y gestionado por la UNaM, al que Aníbal ingresó como becario y representante estudiantil. En ese tiempo, además, contó con una ayuda económica otorgada por una fundación internacional con sede en Holanda, que opera a partir de relaciones con ONGs locales en distintas regiones de Argentina, Perú, Bolivia y Colombia.

Este breve lapso de holgura le permitió dedicarse junto a su hermano a fortalecer la formación de los estudiantes de la escuela primaria en su comunidad.

Los fines de semana íbamos a la comunidad para dar como tutorías, la idea surgió de nosotros porque nuestros sobrinitos tenían problemas para estudiar y no querían ir a la escuela. Y queríamos tratar de ayudar en lo que podíamos, ayudar en el rendimiento.

La fragilidad de este entramado consecutivo de ayudas económicas que involucra a diversas instituciones y agentes municipales, provinciales, nacionales e internacionales, se pone en evidencia para Aníbal cuando finalmente le surge una oferta laboral en el rubro para el que se está formando. Hacia mediados de 2017 le ofrecen un puesto como monitor pedagógico en una institución educativa de nivel medio con un contrato sin relación de dependencia por cinco meses. Para acceder al mismo debía inscribirse en Rentas y la Administración Federal de Ingresos Públicos (AFIP) a fin de pagar los impuestos correspondientes. A raíz de su computación en el sistema tributario nacional, aún en la escala salarial más baja, su familia dejó de percibir la $\mathrm{AUH}^{21}$. Simultáneamente, la institución educativa demoró el pago de su salario, por lo que no le quedó otra alternativa

20- Programa de Respaldo a Estudiantes de Argentina (PROGRESAR) es un programa estatal integral de Becas Educativas que acompaña al alumno en todos los niveles de formación durante su trayectoria académica, a través de un incentivo económico y un importante estímulo personal, que le permitirá poder avanzar en sus estudios hasta completarlos.

21- Asignación Universal por Hijo es una asignación económica mensual por hasta cinco hijos menores de 18 años. La cobra uno solo de los padres, con prioridad para la madre. Para percibir este beneficio, el/la titular debe presentar mensualmente la libreta escolar y sanitaria de cada hijo/a. Corresponde esta asignación a padres que vivan con sus hijos menores en calidad/situación de: desocupado, trabajador del servicio doméstico, trabajador no registrado, monotributista social o inscritos en programas nacionales de trabajo como Hacemos futuro o Manos a la obra. 
que renunciar al empleo tres meses después de iniciado; no podía hacer frente al pago de impuestos (rentas y monotributo), y tuvo que volver a solicitar en ANSES el alta de su mujer en la AUH.

Actualmente, a Aníbal le falta una materia y la presentación de una tesina para finalizar su carrera de grado. De hacerlo, sería el primer estudiante guaraní egresado de la Universidad Nacional de Misiones en sus más de 45 años de existencia. Para terminar su carrera, tiene la opción de presentar una monografía producto de una investigación o un proyecto de intervención social. Aunque la mayoría de sus compañeros decantaron por la primera opción, Aníbal eligió realizar una propuesta de intervención en la escuela primaria intercultural ubicada en su comunidad, donde tuvo el primer contacto con la instrucción formal. Esta opción denota un compromiso con la comunidad a partir de la centralidad otorgada al parentesco, el territorio y la identidad étnica en el modo de organización guaraní. Mientras que la vinculación con la educación está dada a raíz de un proceso de reflexividad ejercida sobre su propia trayectoria como estudiante indígena en el sistema educativo de la sociedad dominante.

\section{Alex, estudiante guaraní de Paraná}

Alex tiene 29 años y estudia Administración en la Universidade Estadual de Londrina. Nació en una aldeia indígena ubicada en el interior del estado de Paraná, en la que su padre ejerce el rol de txamói (pajé o rezador), un liderazgo político y religioso. Alex tiene 13 hermanos, uno de los cuales está siendo preparado por su padre para que eventualmente lo reemplace, mientras que otros tres también estudian en la universidad. Como Crispín y Aníbal, el primer contacto de Alex con la educación formal (Ensino Fundamental) fue posible debido a que la comunidad contaba con una escuela en su territorio.

A minha trajetória na escola da aldeia foi marcada pela liberdade junto às outras crianças Guarani, amigos e parentes, sendo esse ambiente familiar para mim, já que me sentia à vontade com as brincadeiras e o modo de vida de minha comunidade... Contudo, a escolarização na aldeia foi limitada, não me preparando para acompanhar os estudos na escola da cidade, pois considero o ensino da aldeia limitado, muito diferente da escola "lá fora" (na escola da cidade). (CASSANDRE; AMARAL; SILVA, 2016, p. 942).

Para continuar sus estudios Alex tuvo que trasladarse fuera de la comunidad, en dirección a la ciudad capital de Paraná, Curitiba, donde consigue terminar su educación inicial en un programa educativo para jóvenes y adultos, debido al desfasaje de su edad con respecto a los otros alumnos primarios. Posteriormente, en esa misma institución educativa, completa los restantes niveles educativos básicos (Ensino Médio), en modalidad regular. Su migración a la ciudad fue acompañada por su hermano, lo cual aliviano la experiencia de desarraigo, un sentimiento que lo acompañará durante toda su trayectoria educativa. De esa época, recuerda la buena predisposición de los profesores, no así de sus demás compañeros no indígenas, quienes "[...] agiam com 
preconceito, questionando minha presença, insinuando que meu lugar era na aldeia e não ali” (CASSANDRE; AMARAL; SILVA, 2016, p. 943).

Aunque no se dejó desalentar por estas insinuaciones, considera que así como la educación brindada en la comunidad le resultó insuficiente para afrontar la enseñanza media, de la misma manera ésta es limitada en relación a la exigencia de la vida universitaria. Hacia el final de su enseñanza media asistió a una charla sobre marketing y plan de negocios que lo incentivó a seguir la carrera de Administración. Aunque en ese momento no tenía una clara idea acerca de qué consistía esta disciplina, pensó que “[...] poderia fazer uso do Plano de Negócios com intenção de divulgar para a sociedade quem são os índios, nossa cultura aos que quisessem conhecê-la, contribuindo para a comercialização do artesanato" (p. 943).

Como mencionamos anteriormente, el ingreso de estudiante indígenas en IES en Brasil como en Argentina es un fenómeno reciente. Paraná cuenta para esto con una ley estatal novedosa que constituye una experiencia clave a nivel nacional, que garantiza el ingreso de miembros de estas poblaciones a través de un sistema de cupos en la Educación Superior. Alex aprovechó este recurso para iniciar en 2008 sus estudios universitarios en una institución de administración estatal. No obstante, la falta de familiaridad con la experiencia académica adopta en las trayectorias de estos estudiantes matices que suelen pasar desapercibidos para sus colegas no indígenas.

Minha decisão de ingressar na universidade foi motivada pelo desafio em demonstrar que o índio tem capacidade de entrar na universidade para conquistar as coisas que quer, mas na realidade não é assim. Não foi como eu havia pensado, pois tive muitas dificuldades, já que as matérias são muito complexas para a minha realidade. Minhas principais dificuldades foram a de me associar com o meio acadêmico, pois eu não tinha informações de como procurar os recursos para acompanhar as atividades, como o computador e a internet, recursos esses que nunca tinha acessado. Tive de me adaptar às práticas dos não índios... Além disso, não tinha uma noção do que era o curso, diferentemente dos meus colegas que já tinham clareza e informação sobre o funcionamento do curso e da universidade. (p. 943).

El cambio drástico en las modalidades de interacción, los conocimientos básicos y tácitos que se demandan en el ambiente universitario, así como la experiencia de desarraigo de la comunidad, son causas recurrentes de deserción entre estudiantes indígenas, que en las IES del estado de Paraná alcanzó en 2013 la elevada cifra de 43,9\% (CASSANDRE; AMARAL; SILVA, 2016). Este dato es indicativo de que las políticas públicas que garantizan el ingreso de población indígena a la universidad, aunque claves, resultan insuficientes para aumentar los niveles de retención y egreso. A esto se agrega la recurrente falta de acompañamiento durante su tránsito por IES. Para Alex, esta falta de acompañamiento estaba asociada a que los profesores "[...] acham que todo mundo é igual; ser índio e passar no vestibular pela seleção específica indígena é um diferencial, mas depois todos, na universidade, são tratados como iguais, não sendo reconhecidas nossas diferenças" (CASSANDRE; AMARAL; SILVA, 2016, p. 943). A pesar de que el portugués constituye la lengua materna de su aldea, siendo el guaraní un idioma transmitido exclusivamente 
por los abuelos y padres, aunque no hablado con regularidad en espacios informales de la vida comunitaria, Alex se encontró con muchas dificultades para realizar las tareas asignadas durante su cursado, principalmente debido a su falta de familiaridad con el lenguaje académico. Este extrañamiento era reconocido por los demás estudiantes, quienes a menudo pensaban que su colega indígena era extranjero, posiblemente peruano, paraguayo o boliviano. Para superar estas instancias contó con la ayuda invaluable de un compañero no indígena, quien no solo lo asistió en la preparación de trabajos y exámenes, sino que además le procuró los materiales de estudio, ya que los recursos económicos de Alex eran sumamente limitados.

Debido a la escasez de dinero, Alex tampoco podía volver regularmente a su comunidad, tornando la experiencia universitaria más disruptiva. Aunque al inicio vivía con su hermana, que estudiaba Letras en la misma universidad, la ruptura con su forma de vida comunitaria fue una de las principales dificultades de Alex para integrarse en la vida académica. Motivado a restablecer parcialmente su estilo de vida anterior, Alex decidió mudarse a la residencia ubicada en un centro cultural indígena del municipio y casarse con una estudiante guaraní que estudiaba Medicina en su misma universidad. Según sus palabras, "Nossa união foi motivada pela manutenção dos costumes Guarani e pela segurança de viver com uma pessoa que também seja Guarani” (p. 942).

Los obstáculos de asimilación, sin embargo, no finalizaron allí, a veces adoptando un cariz hostil por parte de otros estudiantes y profesores. Su esposa llegó incluso a escuchar de una profesora que los indígenas eran unos "caras de pau" (caraduras, sinvergüenzas) porque, aunque contaban con becas de apoyo, aun así, no aprobaban las materias. Aunque estas becas son fundamentales para garantizar la permanencia de indigenas en las universidades estatales, a menudo resultan insuficientes, y los estudiantes conviven con una presión económica constante.

Mediando su carrera, Alex y su esposa deciden pedir el traslado de la primera universidad en que estudiaban -Universidade Estadual de Maringá- hacia su actual casa de estudios, la Universidade Estadual de Londrina. Allí, la presión económica de la pareja pudo ser aliviada gracias a que Alex consiguió trabajo en una carnicería, gracias a la mediación de un estudiante no indígena. Sus ingresos salariales le permitieron además ayudar con la economía de su familia, disminuyendo la tensión que provocaba en él la inserción en la vida mercantil del mundo no indígena.

Nesse momento comecei a me manter sozinho, não parecia ser um indígena, não me senti indígena, pois não nos consideram apegados ao dinheiro; eu ganhava bolsa e ganhava salário, então pensei: preciso de dinheiro, o necessário, não viver pelo dinheiro. Por outro lado, não senti vergonha porque estava ajudando financeiramente minha família. (p. 944).

Fue a partir de este contacto con el mercado laboral de la sociedad dominante que Alex comenzó a idear la posibilidad de establecer un frigorífico o carnicería dentro o cerca de su comunidad cuando finalice sus estudios. De esta manera, podría generar empleo para la zona. No obstante, manifiesta que si tuviera la oportunidad de incorporarse como servidor público en alguna dependencia pública, no dudaría en 
aceptar. Alex considera que es a partir de la inserción de profesionales indígenas en las instituciones de servicios públicos, particularmente aquellas con injerencia directa sobre las comunidades, como se pueden comenzar a gestar cambios para mejorar la vida en su aldea y para su grupo étnico, pero este constituye apenas un paso, ya que "[...] ainda que o indígena fosse empregado na aldeia, ele não tem condições de provocar mudanças profundas nos serviços públicos na aldeia, pois o profissional que atende na comunidade precisa responder a uma instituição e a um chefe” (p. 944). Desde el punto de vista de Alex, y de otros miembros de su comunidad, la única forma de producir transformaciones sustanciales para su pueblo está en garantizar la representación política de los indígenas en las principales esferas de poder del estado.

Tenho ouvido minha comunidade dizer que é necessário que haja sempre um político representando os indígenas, dando as diretrizes de como fazer e quando fazer. A gente tem de achar um jeito de manter alguém "lá em cima" (nas esferas dos poderes Legislativo, Executivo e Judiciário) e manter "lá embaixo" (na comunidade), porém, não esquecendo que somos indígenas, um povo sofrido que precisa de ajuda. (p. 944).

\section{Reflexiones finales}

Las narrativas presentadas exponen las fragilidades que los estudiantes indígenas experimentan cuando intentan ejercer su derecho a la educación superior. También nos permiten reflexionar sobre la falta de preparación de estas instituciones para recibir y acompañar a estas poblaciones durante su formación. A continuación, evaluaremos al menos cuatro características comunes a los relatos de Aníbal y Alex. Finalmente, analizaremos cómo el tránsito permanente entre la comunidad étnica y el sistema educativo formal ha producido a partir del ingreso en la educación superior la emergencia de un nuevo sujeto social indígena, caracterizado por una doble pertenencia: académica y guaraní. Así como los cambios que estos sujetos tienen el potencial de producir en la mediación entre instituciones estatales y poblaciones originarias (CASSANDRE; AMARAL; SILVA, 2016).

En primer lugar, hay que resaltar el hecho de que los dos estudiantes provienen de familias directamente vinculadas a posiciones de poder político y/o religioso en sus comunidades de origen. Esta característica no solo es fundamental para entender las motivaciones simbólicas y materiales que sus parientes les proveyeron para continuar sus estudios, sino sobre todo en su dinámica de inserción en la educación superior. Al igual que Aníbal,

Alex vem de uma família de lideranças que, assim como outras famílias guarani e kaingang, preparam estrategicamente seus filhos para ocupar a universidade (AMARAL, 2010). Assim, a universidade acaba sendo uma estratégia de manutenção do status de liderança na terra indígena. (CASSANDRE; AMARAL; SILVA, 2016, p. 945).

Una vez que acceden al sistema educativo superior, adquieren al interior de sus comunidades una distinción simbólica que los posiciona por encima de la media 
a la hora de influir en la vida política y de relacionarse con agentes e instituciones estatales con injerencia en esfera comunitaria.

Un segundo rasgo compartido en sus trayectorias está dado por su acceso a la educación formal en el ámbito comunitario, a través de la escuela de modalidad EIB en Misiones y la escola indigena en Paraná. Este primer contacto con la instrucción formal de la sociedad dominante al interior de las comunidades va a jugar un papel formativo en sus trayectorias, prefigurando cómo estos estudiantes van a interpretar luego sus posteriores experiencias educativas. En los dos casos hay una coincidencia en remarcar el ámbito de aprendizaje como familiar y distendido, en contraposición a sus restantes experiencias escolares. No obstante, también coinciden en señalar que la calidad de la instrucción impartida resulta deficiente para afrontar los subsiguientes niveles, volviéndose un obstáculo acumulativo en su desempeño académico.

Una tercera similitud es la necesidad de migración fuera de sus comunidades para continuar su formación. En el caso de Aníbal, el desarraigo adopta características más traumáticas debido a las presiones económicas constantes de sus unidades domésticas, en especial a partir de que conforma su propia familia. Conjugadas, estas características volvieron su derrotero educativo discontinuo, teniendo que interrumpir en sucesivas ocasiones la asistencia escolar y cambiar varias veces de lugar de residencia. En este decurso, los dos estudiantes manifiestan dificultad para mantener un contacto regular con sus familias y sus comunidades, volviendo la distancia espacial disruptiva de la dinámica comunitaria guaraní $\mathrm{y}$, por ende, haciendo problemática su inserción en las instituciones educativas y en la vida urbana. A fin de sobrellevar los traslados constantes, tanto Alex como Aníbal recurrieron a la movilidad conjunta con parientes como estrategia de adaptación al nuevo medio. Más aún, en el caso de Alex esta necesidad de reafirmar la identidad étnica una vez fuera de la comunidad, se traduce durante su inserción a la universidad en una estrategia matrimonial para preservar su modo de vida guaraní. También se percibe a partir de esta instancia el efecto acumulativo de la deficiente formación previa y la interrupción en los estudios, teniendo que recurrir a modalidades aceleradas de cursado debido al desfasaje en la edad.

Por último, la tensión entre la pertenencia étnica y la inserción en ámbitos educativos formales distintos del comunitario, acciona en los dos casos una forma de afirmación de la identidad indígena a partir de generar modalidades de intervención que permitan mejorar las condiciones de vida en las comunidades, sea a través de un emprendimiento que traerá trabajo (Alex), o como tutor/trabajador social (Aníbal).

Como portadores de elementos históricos, culturales, sociales, cosmológicos, lingüísticos y políticos distintos de los que comparte la mayoría de la población universitaria, los estudiantes guaraníes exponen algunas carencias de estas instituciones, cristalizadas en preconceptos, prácticas y estrategias pedagógicas estandarizadas, indicando que están mal dotadas para albergar la diferencia. En este sentido, la dimensión colectiva y comunitaria de las representaciones y prácticas académicas y profesionales que emergen de las narrativas presentadas pone en evidencia el valor central que las universidades otorgan a la acumulación de capitales económico y cultural a través del mérito individual (CASSANDRE; AMARAL; SILVA, 2016). Entonces, cabe preguntarse ¿Qué sucede con el 
capital intercultural incorporado por los estudiantes guaraníes en el campo educativo de nivel superior?; o en otras palabras, ¿Cuándo éste capital es apreciado y cuándo deja de ser valioso? En relación con estos interrogantes, observamos que el capital intercultural incorporado por los estudiantes guaraníes es puesto en juego a través de su incorporación en proyectos de extensión e investigación (en los que realizan tareas de transcripción de audios, subtitulado, traducción, de nexo para acceder a las comunidades guaraníes, entre otras) o programas de apoyo destinados a estudiantes de pueblos originarios. Asimismo, les permite acceder al mercado laboral en puestos donde este capital es altamente valorado, por ejemplo el Instituto Nacional de Asuntos Indígenas (INAI), el Ministerio de Educación (como ADIs), el Ministerio de Salud (como agentes sanitarios o en el hospital, incluso siendo aún estudiantes, como traductores cuando se presentan casos de pacientes guaraníes o provenientes del Paraguay), por mencionar algunos. Sin embargo, los programas y planes de estudio de las diferentes carreras universitarias y terciarias estudiadas no contemplan la incorporación de saberes guaranies. De este modo, reconocemos que

[...] una característica básica de las relaciones entre guaraníes e IES es la desigualdad de poder entre las partes, y antes que asegurar una inclusión democrática de los indígenas a la sociedad, reproducen las diferencias y asimetrías económicas, sociales y culturales. (NUÑEZ, 2012, p. 63).

A pesar de lo desalentador de este diagnóstico institucional, hay que resaltar que las iniciativas desarrolladas por los y las estudiantes guaraníes en ambos casos han contribuido a producir cambios notables. En especial dentro de sus comunidades, han conseguido alentar a jóvenes en niveles educativos anteriores a finalizar sus estudios secundarios y continuar estudiando una carrera universitaria, y a otras mujeres que nunca habían ido a la ciudad, a embarcarse en migraciones y proyectos impensados.

Las historias de Aníbal y Alex nos permiten apreciar un fenómeno que se viene dando hace pocos años, la emergencia del profesional indigena. Este nuevo sujeto social se caracteriza por una doble pertenencia, étnica y académica, que tensiona determinados aspectos del sentido común académico. Asimismo, la formación de profesionales indígenas en IES ha contribuido a producir un nuevo circuito laboral, incluso antes de egresar de las mismas. Pues, al ser considerados estudiantes universitarios poseen más chances de conseguir un puesto laboral, por ejemplo, como ADI o agente sanitario (las ocupaciones más usuales). Al mismo tiempo, el ingreso de profesionales indígenas en instituciones estatales, con la afirmación de su doble pertenencia (indígena y empleado público), tiene el potencial de alterar las relaciones de poder, las rutinas, las herramientas y los parámetros que las orientan (CASSANDRE; AMARAL; SILVA, 2016), produciendo en el mediano y largo plazo modificaciones en las perspectivas que orientan los diseños de políticas públicas para estas poblaciones, en especial en relación a la educación formal. 


\section{Referencias}

AMARAL, Wagner Roberto do. As trajetórias dos estudantes indígenas nas universidades estaduais do Paraná: sujeitos e pertencimentos. Curitiba: UFPR, 2010. 591 p. Tese (Doutorado) - Faculdade de Educação, Universidade Federal do Paraná, Curitiba, 2010.

ARCE, Hugo. Estrategias y alianzas en el proceso de escolarización guaraní de Misiones, Argentina. In: PALADINO, Mariana; GARCÍA, Stella Maris (comp.) La escolarización en los pueblos indígenas americanos: impactos y desafíos. Quito: Abya Yala, 2011. p. 63-88.

ARGENTINA. INDEC. Instituto Nacional de Estadística y Censos. Encuesta Complementaria de Pueblos Indígenas (ECPI) 2004-2005: complementaria del Censo Nacional de Población, Hogares y Viviendas 2001. Buenos Aires: Indec, 2004/2005. Disponible en: https://www.indec.gob.ar/micro_sitios/webcenso/ ECPI/pueblos/ampliada_index_nacionales.asp?mode=00. Acceso en: 12 my. 2020.

BERGAMASCHI, Maria Aparecida.; BARCELOS DOEBBER, Michele; OLIVEIRA BRITO, Patricia. Estudantes indígenas em universidades brasileiras: um estudo das políticas de acesso e permanência. Revista Brasileira de Estudos Pedagógicos, Brasília, DF, v. 99, n. 251, p. 37-53, jan./abr. 2018.

BRASIL. IBGE. Instituto Brasileiro de Geografia e Estatística. Censo demográfico 2010. Brasília, DF: IBGE, 2010.

CASSANDRE, Marcio Pascoal; AMARAL, Wagner Roberto do; SILVA, Alexandro da. Eu, Alex, da etnia Guarani: 0 testemunho de um estudante indígena de administração e seu duplo pertencimento. Caderno EBAPE. BR, Rio de Janeiro, v. 14, n. 4, p. 934-947, dez. 2016. Disponible en: http://www.scielo.br/scielo. php?script=sci_arttext\&pid=S1679-39512016000400934\&lng=pt\&nrm=iso. Acceso en: 12 my. 2020.

EMGC. Equipo Mapa Guaraní Continental. Cuaderno Mapa Guaraní Continental: pueblos Guaraníes en Argentina, Bolivia, Brasil y Paraguay. Campo Grande: Mundial, 2016.

FAUSTINO, Rosangela Cecilia. Educação e religião Guarani no Paraná: estudo a partir do ritual Nimongarai. Práxis Educativa, Ponta Grossa, v. 7, n. esp., p. 239-263, 2012.

GOROSITO KARMER, Ana María. Liderazgos guaraníes: breve revisión histórica y nuevas notas sobre la cuestión. Avá, Misiones, n. 9, p. 11-27, 2006.

LARRICQ, Marcelo. Ipytuma: construcción de la persona entre los Mbya-Guaraní. Posadas: UNaM, 1993.

MELIÀ, Bartomeu. Educación Indígena y alfabetización. Asunción: Cepag, 2008.

NEUFELD, María Rosa. Investigación antropológica y problemáticas socioeducativas en América Latina. Tendencias y propuestas. In: GARBULSKY, Edgardo (comp.). CONGRESO LATINOAMERICANO DE ANTROPOLOGÍA, 1., 2005, Rosario. Actas del... Rosario: Universidad Nacional de Rosario, 2005. p. 315-326.

NOVARO, Gabriela. Intersecciones entre la investigación y la gestión: avances en el campo de la antropología y la educación. Publicar - En Antropología y Ciencias Sociales, Buenos Aires, v. 8, n. 9, p. 47-71, 2010. 
NUÑEZ, Yamila Irupé. Educación superior e interculturalidad en Misiones - Argentina. Textos \& Debates, Roraima, n. 21, p. 59-74, 2012.

PÖLLMANN, Andreas. Habitus, reflexividad y la realización de capital intercultural: el potencial (no aprovechado) de la educación intercultural. Cultura y Representaciones Sociales, México, DC, v. 11, p. 55-78, 2016a.

PÖLLMANN, Andreas. Capital intercultural. In: CENTER for Intercultural Dialogue (ed.). Key concepts of intercultural dialogue. [S. I.: s. n.], 2016b. Disponible en: https://centerforinterculturaldialogue.files. wordpress.com/2016/07/kc6-intercultural-capital_spanish.pdf. Acceso en: 12 my. 2020.

ROCKWELL, Elsie. La experiencia etnográfica: historia y cultura en los procesos educativos. Buenos Aires: Paidós, 2009.

RUIZ DE MONTOYA, Antonio. [1639] Tesoro de la Lengua Guarani. Edición de Christian F. Seybold. Leipzig: B.G. Teubner, 1876.

Recibido en:27.08.2019

Revisado en: 18.02 .2020

Aprobado en: 12.05 .2020

Yamila Irupé Nuñez es doctora en Antropología Social. Investigadora en la Secretaría de Investigación de la FHyCS-UNaM. Actualmente lleva adelante el proyecto de investigación postdoctoral "Conformación de liderazgos femeninos mbya-guaraní en Misiones, Argentina. Género, trabajo y educación" a través del Consejo Nacional de Investigaciones Científicas y Técnicas (CONICET). 